\title{
Contemplation on Alizarin Blue - A DFT Treatment
}

\section{Lemi Türker}

Department of Chemistry, Middle East Technical University, Üniversiteler, Eskişehir Yolu No: 1, 06800 Çankaya/Ankara, Turkey; e-mail: lturker@gmail.com; lturker@metu.edu.tr

\begin{abstract}
Primarily Alizarin blue is an important acid-base indicator, exhibiting color change at different $\mathrm{pH}$ values in the acid and also base ranges. It is also used as microbiological staining agent. It is in theory capable of forming various tautomers and zwitterions. In the present study, within restrictions of density functional theory at the level of B3LYP/6$31 \mathrm{G}(\mathrm{d})$, some tautomeric and zwitterionic forms of it have been investigated. Some physicochemical, structural properties as well as stabilities, energies, IR and UV-VIS spectra are considered.
\end{abstract}

\section{Introduction}

Alizarin blue (Alizarin blue R) is the common name for 5,6-dihydroxynaphtho[2,3f]quinoline-7,12-dione (or 7.8-dihydroxy-5.6-phthalyquinoline) [1]. It is also named as Alizarin Blue R; C.I. 67410. It is an anthraquinone type dyestuff. It is prepared from 3-nitroalizarin, glycerol and concentrated sulfuric acid (adapted Skraup synthesis [2]) by Auerbach in 1879 [1, 3]. Alizarin blue crystallizes in brownish-violet needles from a benzene solution, (mp. $268-270^{\circ} \mathrm{C}$ ). Its molecular formula is $\mathrm{C}_{17} \mathrm{H}_{9} \mathrm{NO}_{4}$ and has the molecular weight of 291.26 [4]. It could be considered as the derivative of 1,2dihydroxyanthraquinone (Mordant Red 11). It is soluble in glacial acetic acid, amyl alcohol and hot benzene and slightly soluble in ethanol, ether and cold benzene, insoluble in water.

\section{Received: February 10, 2019; Accepted: February 26, 2019}

Keywords and phrases: Alizarin blue, Alizarin blue R, tautomerism, dye, DFT.

Copyright (C) 2019 Lemi Türker. This is an open access article distributed under the Creative Commons Attribution License, which permits unrestricted use, distribution, and reproduction in any medium, provided the original work is properly cited. 

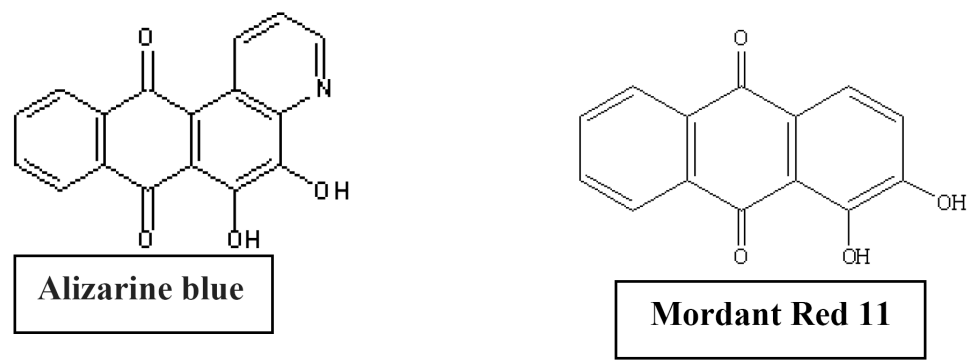

When prepared in a saturated alcoholic solution, Alizarin blue is used as an acidbase indicator. It changes from pink at $\mathrm{pH} 0.0$ to yellow at $\mathrm{pH} 2.6$. It also changes from yellow at $\mathrm{pH} 6.0$ to green at $\mathrm{pH} 7.6$ [5]. Alizarin blue with monosodium sulphite (1:2) is known as C.I.Mordant Blue 27, Alizarin blue BS [6].

A notable use of Alizarin blue is as a staining agent in biological research because it stains free calcium and certain calcium compounds ... Alizarin blue has also some specific usages such as a selective reagent for traces of copper [7].

On the other hand, tautomerism which plays an important role on some properties of molecules should be present in Alizarin blue structure. It is noteworthy that substances which are isomeric under certain given conditions are tautomeric under more drastic conditions [8]. The proton tautomerism is a special kind of cationotropic tautomeric transformations.

In the present study, some tautomeric and zwitterionic structures of Alizarin blue are considered within the constraints of density functional theory (DFT).

\section{Method of Calculations}

The geometry optimizations of all the structures presently considered, leading to energy minima were achieved first by using MM2 method $[9,10]$ for the equilibrium conformer. Subsequent optimizations were achieved at Hartree-Fock level using various basis sets hierarchically. Then, the geometry optimizations were managed within the framework of density functional theory $[11,12]$, finally at the levels of RB3LYP /6$31 \mathrm{G}(\mathrm{d})$. Note that the exchange term of B3LYP consists of hybrid Hartree-Fock and local spin density (LSD) exchange functions with Becke's gradient correlation to LSD exchange $[12,13]$. Note that the correlation term of B3LYP consists of the Vosko, Wilk, Nusair (VWN3) local correlation functional [14] and Lee, Yang, Parr (LYP) correlation correction functional [15]. The vibrational analyses have also been done. The total 
electronic energies are corrected for the zero point vibrational energy (ZPE). The stationary points to energy minima were proved in all the cases by calculation of the second derivatives of energy with respect to the atom coordinates. The normal mode analysis for each structure yielded no imaginary frequencies for the $3 N-6$ vibrational degrees of freedom, where $N$ is the number of atoms in the system. This indicates that the structure of each molecule corresponds to at least a local minimum on the potential energy surface. All these calculations were done by using the Spartan 06 package program [16].

\section{Results and Discussion}

Tautomers having different structures possess dual reactivity, depending on its tautomer content (allelotropic mixture [8]). Note that substances which are isomeric under certain conditions are tautomeric under more drastic conditions $[8,17]$.

Alizarin blue contains two phenolic hydroxy groups which might exhibit keto-enol type tautomerism, even it may spread over the quinoid carbonyl groups. The presence of aza nitrogen of the embedded pyridine ring raises the possibility of zwitterionic structures for Alizarin blue.

In the present study the structures considered are tautomers (A1-A4, see Figure 1) and zwitterions (Z1, Z2, see Figure 2). The tautomers are 1,3- and /or 1,5-type proton tautomers. The zwitterion, $\mathrm{Z} 2$ resembles as 1,5-type tautomer derived from A1 but there is no accompanied shift of electrons to migration of proton.<smiles></smiles><smiles>O=C1C2=c3cccnc3=C(O)C(=O)C2C(=O)c2ccccc21</smiles>

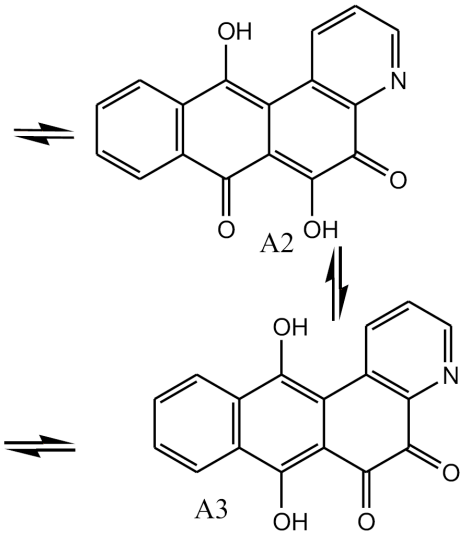

Figure 1. Relationship among the tautomers considered. 
<smiles></smiles><smiles></smiles>

Figure 2. The zwitterions considered.

Figure 3 shows the optimized structures of the presently considered tautomers and zwitterions. Note that in structure $\mathrm{A} 1$, the conformations of $\mathrm{OH}$ groups suggest certain extent of hydrogen bonding between them and also with the aza-nitrogen atom. However, only in A4 structure, a hydrogen bonding is clearly exhibited with the elongated $\mathrm{O}-\mathrm{H}$ bonding.

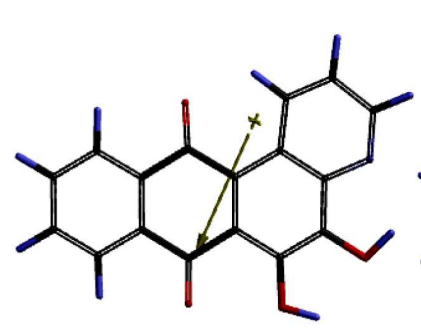

A1

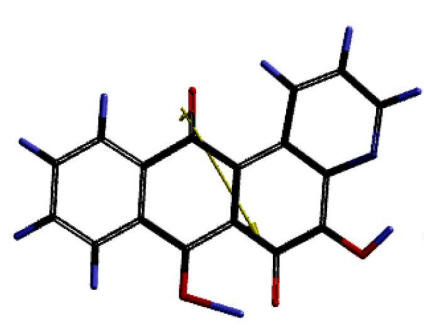

A4

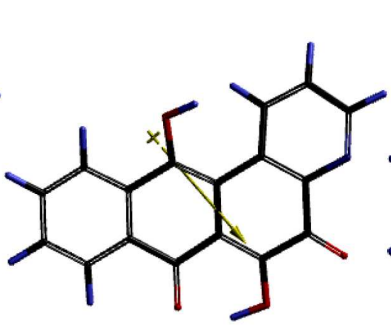

A2

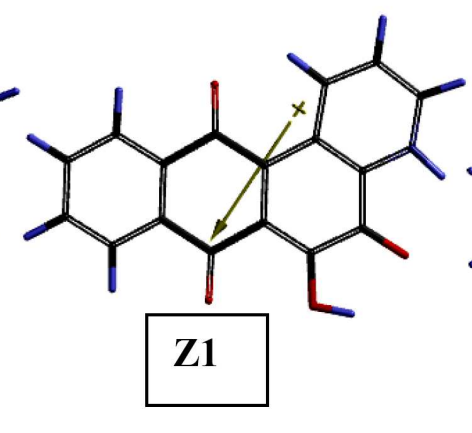

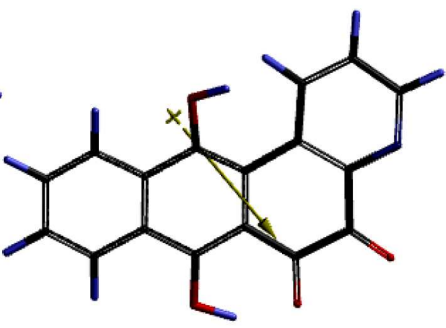

A3

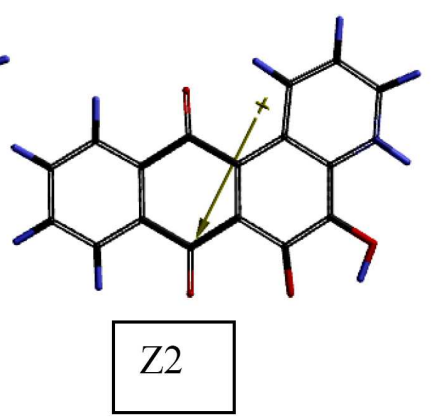

Figure 3. Optimized structures of the species considered.

Table 1 shows some properties of the structures of present concern. All the structures, except A1 and A4 are characterized with dipole moment values in the range 
of 6-8 Debye whereas A1 and A4 have comparatively low dipole moment values. The direction of the dipole moment vectors can be seen in Figure 3.

Table 1. Some properties of the tautomers considered.

\begin{tabular}{lcccccc}
\hline Property & $\mathbf{A 1}$ & $\mathbf{A 2}$ & $\mathbf{A 3}$ & $\mathbf{A 4}$ & $\mathbf{Z 1}$ & $\mathbf{Z 2}$ \\
\hline Area $(\AA 2)$ & 270.09 & 268.48 & 268.65 & 268.13 & 270.20 & 271.10 \\
Volume $(\AA 3)$ & 269.43 & 268.43 & 268.63 & 268.30 & 269.46 & 269.86 \\
$\begin{array}{l}\text { Dipole moment } \\
\text { (Debye) }\end{array}$ & 2.40 & 7.66 & 7.30 & 3.10 & 6.75 & 7.12 \\
$\begin{array}{l}\text { Ovality } \\
\text { Log P }\end{array}$ & 1.34 & 1.33 & 1.33 & 1.33 & 1.34 & 1.34 \\
$\begin{array}{l}\text { Polarizability } \\
\begin{array}{l}\text { Types of } \\
\text { tautomerism } \\
\text { present }\end{array}\end{array}$ & 1.72 & -1.22 & 1.12 & -1.22 & - & - \\
\hline
\end{tabular}

Ovality is a measure of how the shape of the molecule approaches a sphere or cigar and it is described by the ratio of volume and area [16].

$$
\mathrm{O}=\mathrm{A} /\left(4 \pi(3 \mathrm{~V} / 4 \pi)^{2 / 3}\right)
$$

whereas polarizability is defined as [16]

$$
\mathrm{P}=0.08 \mathrm{VdW} \text { Volume }-13.0352 \text { Hardness }+0.97992 \text { Hardnes }^{2}+41.3791
$$

where

$$
\begin{aligned}
& \text { Hardness }=-\left(\varepsilon_{\mathrm{HOMO}}-\varepsilon_{\mathrm{LUMO}}\right) / 2 \\
& \text { Electronegativity }=-\left(\varepsilon_{\mathrm{HOMO}}+\varepsilon_{\mathrm{LUMO}}\right) / 2 .
\end{aligned}
$$

Note that the tautomeric shift occurring in these structures are 1,5- or 1,7-types and the possibility of tautomerism in general follows the order of $1,3>1,5>1,7$ [8].

Figure 4 depicts the IR spectra of the structures considered. The peaks in the region of $1750-1700 \mathrm{~cm}^{-1}$ stand for carbonyl $\mathrm{C}=\mathrm{O}$ stretchings. In the case of zwitterionic species the range for the carbonyl stretchings extends down to $1640 \mathrm{~cm}^{-1}$ due to some unsaturated carbonyl formation possibilities. The sharp peak existing in the spectra of all and occurring about $1305-1324 \mathrm{~cm}^{-1}$ stands for O-H bending coupled with some skeletal vibrations. 

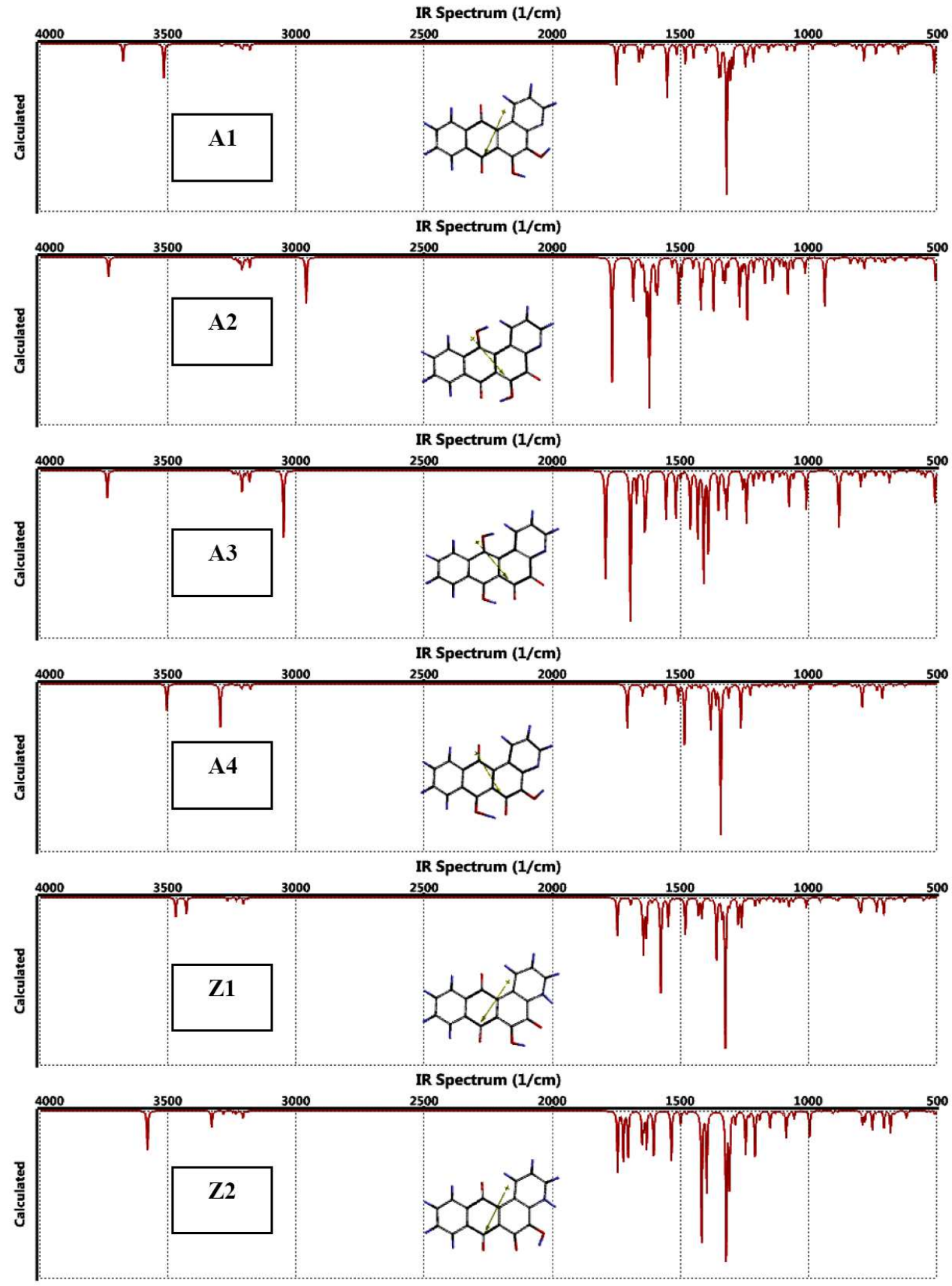

Figure 4. IR spectra of the structures considered. 
Table 2 shows various energies of the structures where $\mathrm{E}$ and ZPE are the total electronic and the zero point vibrational energies, respectively. Energy, abbreviated as $\mathrm{E}_{\mathrm{c}}$, is the total corrected electronic energy. Note that all the structures presented in Table 2 are isomeric in nature. Their $\mathrm{E}_{\mathrm{c}}$ values follow the order of $\mathrm{A} 4<\mathrm{A} 1<\mathrm{Z} 1<\mathrm{A} 3<\mathrm{A} 2<\mathrm{Z} 2$. Noteworthy that A4 is more stable than A1. It should be because of the hydrogen bonding possibility of hydrogen of $\mathrm{OH}$ groups with the nearby keto carbonyl oxygen atom (see Figure 3).

Table 2. Some energies of the structures considered.

\begin{tabular}{|l|c|c|c|}
\hline Structure & $\mathbf{E}$ & $\mathbf{Z P E}$ & $\mathbf{E}_{\mathbf{c}}$ \\
\hline $\mathbf{A 1}$ & -2648846.26 & 588.87 & -2648257.39 \\
\hline $\mathbf{A 2}$ & -2648802.65 & 588.48 & -2648214.17 \\
\hline $\mathbf{A 3}$ & -2648807.45 & 589.04 & -2648218.41 \\
\hline $\mathbf{A 4}$ & -2648884.16 & 590.83 & -2648293.33 \\
\hline $\mathbf{Z 1}$ & -2648828.56 & 590.86 & -2648237.7 \\
\hline $\mathbf{Z 2}$ & -2648798.75 & 588.36 & -2648210.39 \\
\hline
\end{tabular}

Energies in $\mathrm{kJ} / \mathrm{mol}$.

Table 3 tabulates some thermodynamic properties of the structures considered. As the proton moves from one side to other, all of thermodynamic properties change following the order of $\mathrm{A} 4<\mathrm{A} 1<\mathrm{Z} 1<\mathrm{A} 3<\mathrm{A} 2<\mathrm{Z} 2$ and $\mathrm{A} 4<\mathrm{A} 2<\mathrm{A} 3<\mathrm{Z} 1<\mathrm{A} 1<\mathrm{Z} 2$ for $\mathrm{H}^{\circ}$ and $\mathrm{S}^{\circ}$, respectively, whereas, the order of $\mathrm{G}^{\circ}$ is $\mathrm{A} 4<\mathrm{A} 1<\mathrm{Z} 1<\mathrm{A} 3<\mathrm{A} 2<\mathrm{Z} 2$ as same as the order of $\mathrm{H}^{\circ}$ values.

Table 3. Some thermodynamic properties of the structures considered.

\begin{tabular}{|c|c|c|c|}
\hline Structure & $\mathrm{H}^{\circ}$ & $\mathrm{S}^{\circ} * 10^{-3}$ & $\mathrm{G}^{\circ}$ \\
\hline $\mathbf{A 1}$ & -2648242.651 & 481.57 & -2648386.241 \\
\hline $\mathbf{A 2}$ & -2648199.881 & 479.27 & -2648342.762 \\
\hline $\mathbf{A 3}$ & -2648204.082 & 479.30 & -2648346.989 \\
\hline $\mathbf{A 4}$ & -2648279.382 & 477.32 & -2648421.685 \\
\hline $\mathbf{Z 1}$ & -2648223.406 & 479.62 & -2648366.392 \\
\hline $\mathbf{Z 2}$ & -2648195.601 & 482.61 & -2648339.506 \\
\hline
\end{tabular}

Energies in $\mathrm{kJ} / \mathrm{mol}$. 
Table 4 shows $\Delta \mathrm{H}^{\circ}$ and $\Delta \mathrm{G}^{\circ}$ values for the conversion of the structures as indicated in the first column. According to the data A2 $\rightarrow$ A3 and A3 $\rightarrow$ A4 transformations are exothermic and they are thermodynamically feasible, having negative $\Delta \mathrm{G}^{\circ}$ values. All the other transformations should occur in the reverse direction. Hence, the thermodynamic calculations estimate $\mathrm{A} 1 \rightarrow \mathrm{A} 4$ conversion favorable. Also, the stabilities based on $E_{c}$ values (see Table 2) favor A4 tautomer over A1. However, considering the aromaticity of the rings, one observes that $\mathrm{A} 1$ is the unique structure among the tautomers A1-A4 having two Clar's sextets [18] in the embedded quinoline originated moiety (or the maximum number of aromatic sextets).

As for the zwitterionic species Z1 and Z2, the first one is more stable (see Table 2) because in $\mathrm{Z} 2$ the opposite charges are further away from each other compared to the case present in $\mathrm{Z} 1$. Also, $\Delta \mathrm{G}^{\circ}$ values show that the formation of $\mathrm{Z} 2$ is less favorable than Z1.

Figure 5 shows the variation of UV-VIS (time dependent DFT) spectra of the structures as the proton shifts from one site to other.

Table 4. $\Delta \mathrm{H}^{\circ}$ and $\Delta \mathrm{G}^{\circ}$ values for the reactions shown.

\begin{tabular}{ccc}
\hline Conversion & $\mathbf{\Delta H}^{\circ}$ & $\mathbf{\Delta G}^{\mathbf{0}}$ \\
\hline $\mathbf{A 1} \rightarrow \mathbf{A 2}$ & 42.76982 & 43.47871 \\
$\mathbf{A 1} \rightarrow \mathbf{A 3}$ & 38.56898 & 39.25162 \\
$\mathbf{A 2} \rightarrow \mathbf{A 3}$ & -4.20084 & -4.22711 \\
$\mathbf{A 3} \rightarrow \mathbf{A 4}$ & -75.30011 & -74.69620 \\
$\mathbf{A 4} \rightarrow \mathbf{A 1}$ & 36.73111 & 35.44461 \\
$\mathbf{A 1} \rightarrow \mathbf{Z 1}$ & 19.24511 & 19.84898 \\
$\mathbf{A 1} \rightarrow \mathbf{Z 2}$ & 47.04943 & 46.73436 \\
\hline
\end{tabular}

Energies in $\mathrm{kJ} / \mathrm{mol}$.

As seen in the figure, the zwitterionic species have exhibited a bathochromic effect and in that sense they are distinctly different from the other structures. Table 5 shows the $\lambda_{\max }$ values and related intensities of the peaks shown in Figure 5. It also displays the components of the transition moments of the UV-VIS excitations.

Table 6 shows the highest occupied and the lowest unoccupied molecular orbitals (HOMO and LUMO, respectively) energies and also the interfrontier molecular orbital energy gap $\left(\varepsilon_{\mathrm{LUMO}}-\varepsilon_{\mathrm{HOMO}}\right)$ values of the structures. 

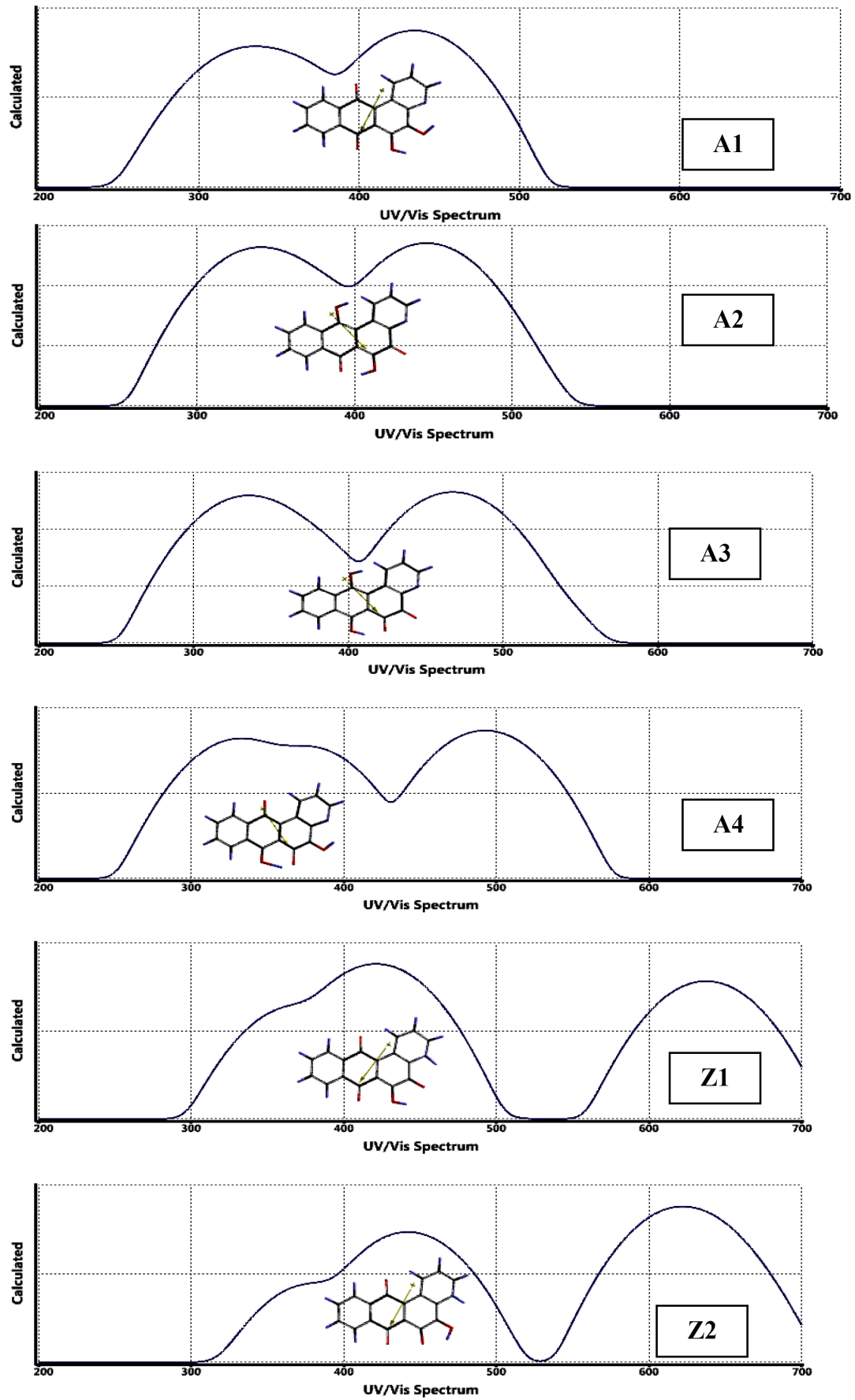

Figure 5. UV-VIS spectra of the tautomers considered. 
Table 5. UV-VIS spectral characteristics of the main bands of the tautomers considered.

Tautomer $\lambda_{\max }(\mathrm{nm}) \quad$ Intensity Component of the transition moment

\begin{tabular}{lccccc}
\hline & & & $\mathbf{X}$ & $\mathbf{Y}$ & $\mathbf{Z}$ \\
\hline $\mathbf{A 1}$ & 434.72 & 0.1716 & 0.7581 & -0.5396 & -1.2612 \\
& 353.68 & 0.017 & 0.1983 & -0.3475 & -0.2179 \\
& 331.29 & 0.0421 & -0.3323 & 0.3705 & 0.4599 \\
\hline $\mathbf{A 2}$ & 445.93 & 0.2675 & -0.6406 & -0.0886 & -1.8734 \\
& 360.77 & 0.0131 & 0.2151 & 0.0336 & 0.3286 \\
& 339.81 & 0.1877 & -0.1917 & 0.0716 & -1.4346 \\
\hline $\mathbf{A 3}$ & 467.77 & 0.2081 & -0.0978 & -0.8133 & -1.5918 \\
& 335.27 & 0.1529 & -0.1490 & 0.6173 & 1.1332 \\
\hline $\mathbf{A 4}$ & 492.73 & 0.1617 & 1.4916 & -0.4750 & -0.4146 \\
& 376.56 & 0.0423 & -0.6793 & 0.0560 & -0.2455 \\
& 332.55 & 0.0822 & -0.8995 & 0.0941 & -0.2862 \\
\hline $\mathbf{Z 1}$ & 637.56 & 0.0504 & 0.5225 & -0.2467 & -0.8512 \\
& 421.32 & 0.1948 & 0.8413 & -0.6416 & -1.2578 \\
\hline $\mathbf{Z 2}$ & 622.15 & 0.1887 & 0.9024 & -1.4095 & -1.0312 \\
& 442.14 & 0.0261 & 0.3180 & -0.2031 & -0.4870 \\
\hline
\end{tabular}

Table 6. The HOMO, LUMO energies and the frontier molecular orbital energy gaps $(\Delta \varepsilon)$ of the tautomers considered.

\begin{tabular}{|c|c|c|c|}
\hline Tautomer & HOMO & LUMO & $\boldsymbol{\Delta} \boldsymbol{\varepsilon}$ \\
\hline $\mathbf{A 1}$ & -575.66 & -280.74 & 294.92 \\
\hline $\mathbf{A 2}$ & -565.42 & -301.74 & 263.68 \\
\hline $\mathbf{A 3}$ & -565.48 & -292.50 & 272.98 \\
\hline $\mathbf{A 4}$ & -560.20 & -306.85 & 253.35 \\
\hline $\mathbf{Z 1}$ & -505.90 & -287.31 & 218.59 \\
\hline $\mathbf{Z 2}$ & -503.66 & -298.47 & 205.19 \\
\hline
\end{tabular}

Energies in $\mathrm{kJ} / \mathrm{mol}$.

The HOMO energies follow the order of $\mathrm{A} 1<\mathrm{A} 3<\mathrm{A} 2<\mathrm{A} 4<\mathrm{Z} 1<\mathrm{Z} 2$. The order for the LUMO energies is $\mathrm{A} 4<\mathrm{A} 2<\mathrm{Z} 2<\mathrm{A} 3<\mathrm{Z} 1<\mathrm{A} 1$. Consequently, $\Delta \varepsilon$ values follow the order of $\mathrm{Z} 2<\mathrm{Z} 1<\mathrm{A} 4<\mathrm{A} 2>\mathrm{A} 3<\mathrm{A} 1$. The zwitterionic species have higher HOMO energy level than 
the others. Note that generally electron donating groups rise up the HOMO and LUMO energy levels, whereas electron attractors lower them [19]. In that sense, negatively charged oxygen atom in $\mathrm{Z} 1$ and $\mathrm{Z} 2$ is more effective than the positive charge on the nitrogen atom (protonated). It is because of the fact that the negative charge on the oxygen is directly (mesomerically) participates in the conjugation, whereas the positive charge is localized and influence the system inductively. Also note that the formation of zwitterionic species results in raising of the HOMO energy level. Both of these variations cause the narrowing of interfrontier molecular orbital energy gaps. Thus, the zwitterionic species are characterized with bathochromic shift.

Figure 6 displays some of the molecular orbital energy levels of the structures. As seen there, the HOMO energy level of the tautomers (A1-A4) is comparable to each other. The LUMO energies vary in a larger span. Also, the inner lying molecular orbital energy levels exhibit very different distribution. On the other hand, the zwitterionic species have comparable HOMO and LUMO energies with each other but their NEXTHOMO levels are considerably different.
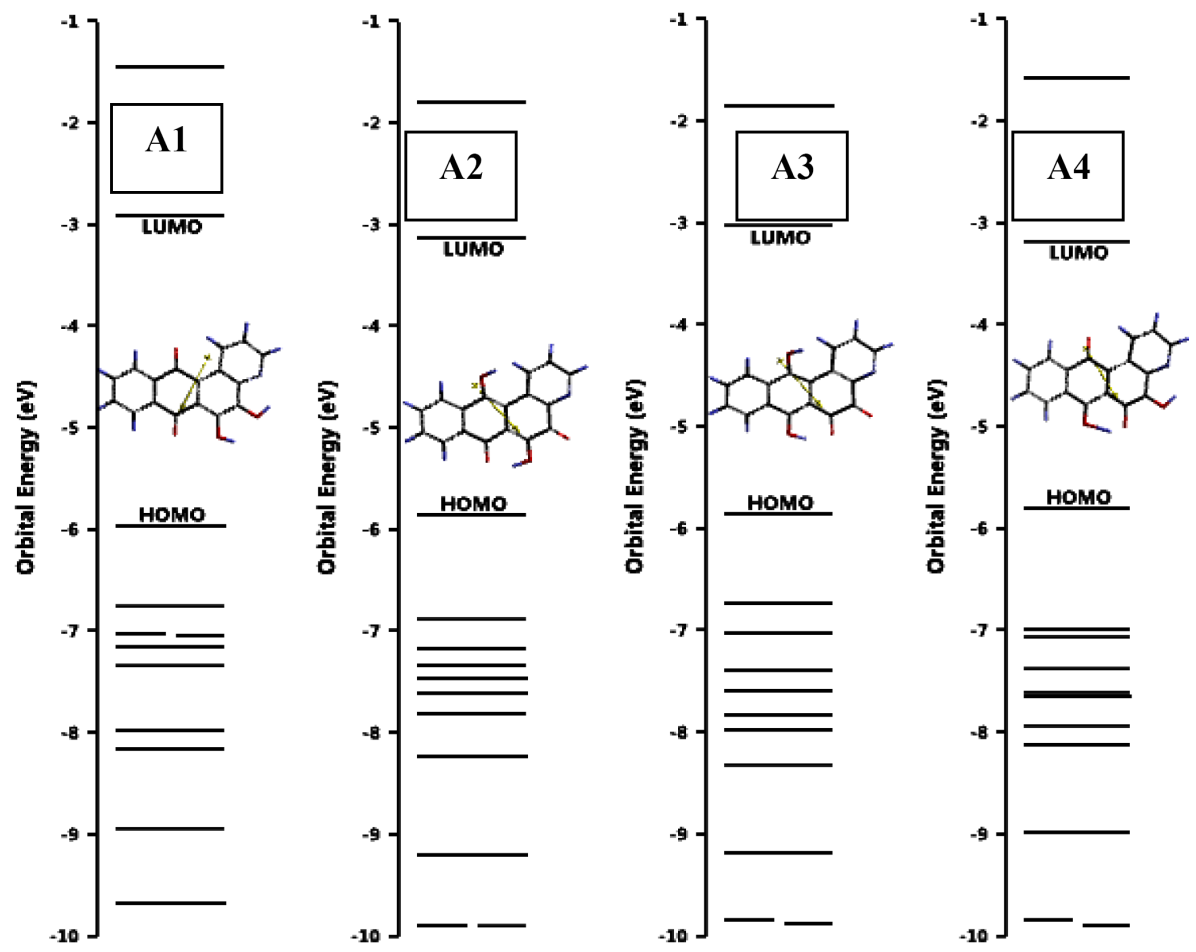

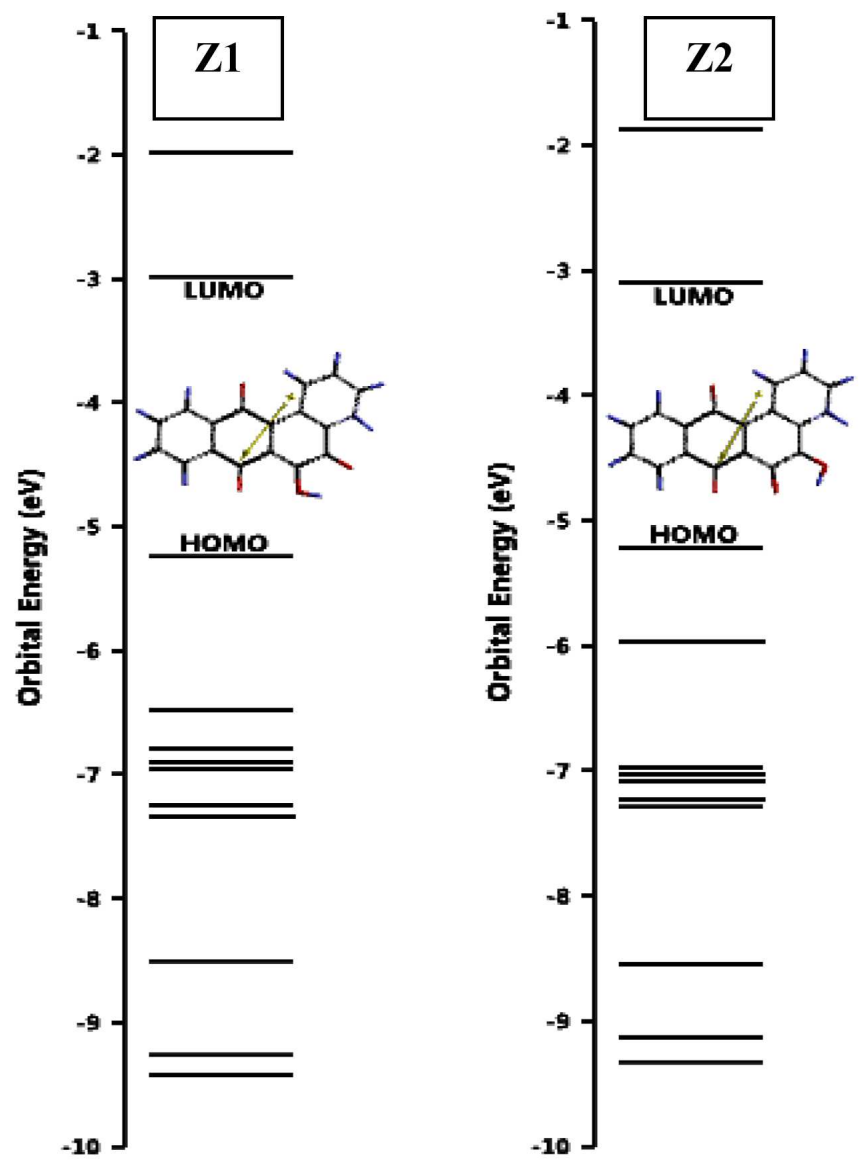

Figure 6. Some of the molecular orbital energy levels of the tautomers considered.

Table 7 shows the solvation energies for the species presently considered. Being a dyestuff, aqueous properties of Alizarin blue has the prime importance for many purposes.

Table 7. Calculated solvation energies for the structures considered.

\begin{tabular}{|l|c|c|c|c|c|c|}
\hline $\begin{array}{l}\text { Solvation } \\
\text { method }\end{array}$ & A1 & A2 & A3 & A4 & Z1 & Z2 \\
\hline SM5.4/A & -38.316 & -69.581 & -63.893 & -56.180 & -72.612 & -64.440 \\
\hline SM50R & -86.91 & -89.63 & -90.95 & -86.77 & -188.55 & -359.57 \\
\hline
\end{tabular}

Energies in $\mathrm{kJ} / \mathrm{mol}$. 
Among the tautomers the calculated solvation energies [16] follow the order of $\mathrm{A} 2<\mathrm{A} 3<\mathrm{A} 4<\mathrm{A} 1$ (SM5.4/A) and A3<A2 $<\mathrm{A} 1<\mathrm{A} 4$ (SM50R). According to the second method A1 is solvated slightly better than A4. In the case of the zwitterionic species (being charge separated structures) the charge-dipole interactions between the solute and water molecules should be more pronounced than the dipole-dipole interactions and they should be hydrated better. In the second case, solvation energies of the zwitterionic species are more negative than the isomeric tautomeric structures. Moreover, Z2 is solvated better than $\mathrm{Z} 1$ although the stability order (in vacuum) is the reverse of it (see Table 2). Note that in $\mathrm{Z} 2$ the charge separated centers are farther away from each other than the case in $\mathrm{Z} 1$, hence $\mathrm{Z} 2$ is more apt to decrease its energy by solvation.

Table 8 shows the energies of the species in water.

Table 8. Energy (aq) values $(\mathrm{kJ} / \mathrm{mol})$.

\begin{tabular}{cccccc}
\hline $\mathbf{A 1}$ & $\mathbf{A 2}$ & $\mathbf{A 3}$ & $\mathbf{A 4}$ & $\mathbf{Z 1}$ & $\mathbf{Z 2}$ \\
\hline-2648884.57 & -2648872.23 & -2648871.34 & -2648940.34 & -2648901.17 & -2648863.19 \\
\hline
\end{tabular}

Note that Cramer-Truhlar solvation method calculates the solvation energy by adding electronic energy and solvation energy and given as "Energy (aq)" for water by the program. The method of SM50R is a solvation method derived from the semiempirical SM54 approach. It is independent of the wavefunction and depends only on the geometry of the molecule [20].

\section{Conclusion}

The present DFT treatment of Alizarin blue has considered various tautomeric and zwitterionic species of it. It has revealed that electronically the most stable structure is A4. Its formation from A1 is also thermodynamically a feasible process, namely A1 $\rightarrow$ A4 is $-35.44 \mathrm{~kJ} / \mathrm{mol}$. However, A1 has more Clar's sextets than A4 has which should be the main contributor to the aromatic stabilization. The zwitterions considered are less stable than A1 and A4 and thermodynamically they tend to transform into A1 structure. A remarkable feature of the zwitterionic species is that they exhibit bathochromic shift into the visible region. One should keep in mind that the present results within the constraints of the level of calculations hold for vacuum conditions. Obviously in aqueous medium the whole picture might be somewhat different. 


\section{References}

[1] M. Windholz (Ed.), The Merck Index, 10th ed., Rahway, NJ: Merck Co. Inc., 1983.

[2] A. I. Vogel, Practical Org. Chem., 3rd ed., London: Longman, 1967.

[3] G. Auerbach, Alizarine blue, Journal of the Chemical Society, Transactions 35 (1879), 799-805.

[4] R. S. Lewis, Hawley's Condensed Chemical Dictionary, 10th ed., New York: Van Nostrand-Reinhold, 1993.

[5] S. Budavari (Ed.), The Merck Index, 12th ed., NJ: Merck Research Labs, 1996.

[6] Ju. Lurie, Handbook of Analytical Chemistry, Moscow: Mir Pub., 1975.

[7] F. Feigl and A. Caldas, Alizarin blue as a selective reagent for traces of copper, Analytica Chimica Acta 8 (1953), 117-121.

[8] O. Reutov, Theoretical Principles of Organic Chemistry, Moscow: Mir Pub., 1970.

[9] A. R. Leach, Molecular Modeling, Essex: Longman, 1997.

[10] P. Fletcher, Practical Methods of Optimization, New York: Wiley, 1990.

[11] W. Kohn and L. Sham, Self-consistent equations including exchange and correlation Effects, J. Phys. Rev. 140 (1965), 1133-1138.

[12] R. G. Parr and W. Yang, Density Functional Theory of Atoms and Molecules, London: Oxford University Press, 1989.

[13] A. D. Becke, Density-functional exchange-energy approximation with correct asymptotic behavior, Phys. Rev. A 38 (1988), 3098-3100.

[14] S. H. Vosko, L. Vilk and M. Nusair, Accurate spin-dependent electron liquid correlation energies for local spin density calculations: a critical analysis, Can. J. Phys. 58 (1980), 1200-1211.

[15] C. Lee, W. Yang and R. G. Parr, Development of the Colle-Salvetti correlation-energy formula into a functional of the electron density, Phys. Rev. B 37(1988), 785-789.

[16] SPARTAN 06, Wavefunction Inc., Irvine CA, USA, 2006.

[17] E. V. Anslyn and D. A. Dougherty, Modern Physical Organic Chemistry, Sausalito, California: University Science Books, 2006.

[18] E. Clar, The Aromatic Sextet, London: Wiley, 1972.

[19] I. Fleming, Frontier Orbitals and Organic Chemical Reactions, NY: Wiley, 1976.

[20] C. J. Cramer and D. G. Truhlar, PM3-SM3: A general parameterization for including aqueous solvation effects in the PM3 molecular orbital model, J. Compt. Chem. 13(9) (1992), 1089-1097. 